\title{
Chinos e identidad panameña
}

\section{Jorge Kam Ríos ${ }^{1, *}$}

1Profesor, Facultad de Humanidades y Ciencias Religiosas, Universidad Santa María La Antigua (USMA), Apartado Postal 0819-08550, Panamá, República de Panamá.

*Autor para correspondencia. Email: jorgekam@gmail.com

Recibido: 5 de abril de 2015

Aceptado: 17 de abril de 2015

\begin{abstract}
The Chinese presence in Panama and its contribution to the development and consolidation of the Panamanian national culture represent areas of intellectual, investigative and speculative work with much validity and relevance. We present here the progress of an ongoing research project on this matter. This historical research project is based upon heuristic-hermeneutic procedures, using the ethnographic sub-method of analysis and strongly based in major criticism of sources (analysis of the contents of documental support sources; comparison and contrast with content from other sources; and analysis of the physical properties of the documental support sources).
\end{abstract}

Keywords: Chinese people, Immigration, Chinese culture, Panamanian culture, Racial discrimination, Panamanian history identity.

\section{Resumen}

La presencia de los chinos en Panamá y su aporte al desarrollo y la consolidación de la cultura nacional panameña representan ámbitos de trabajo intelectual, investigativo y especulativo con mucha vigencia y pertinencia. Presentamos aquí los avances de un trabajo en esta materia, en pleno desarrollo. Este proyecto de investigación histórica se basa en procedimientos heurístico-hermenéuticos, empleando el submétodo etnográfico de análisis y con base en la crítica mayor de fuentes (análisis del contenido de documentos soporte de las fuentes; comparación y contrastación con el contenido de otras fuentes; y análisis de las propiedades físicas de los documentos soporte de las fuentes).

Palabras clave: Pueblo chino, Inmigración, Cultura china, Cultura panameña, Discriminación racial, Historia panameña. 
Invest. pens. crit.

Vol. 3, No. 1, enero-abril 2015.

pp. 39-46

El tema de la presencia de China o de chinos en Panamá es una reivindicación a un pueblo que se instaló, lentamente, en éste territorio, sufriendo una serie de peripecias y atropellos por parte de los naturales del Istmo de Panamá (Chen, 2006).

La cultura nacional, la del Estado, no reconoce otra esencia que la panameña, pero por los espacios donde se desarrollan las culturas en el territorio estatal, los panameños son mayoritariamente de cultura hispanoamericana. Los panameños no pueden ser distinguidos por el color de su piel y profesan la religión cristiana, casi un $80 \%$ en relación a las otras denominaciones o creencias. Los panameños expresan su cultura en castellano y sus danzas y comidas son el producto final de una serie de mezclas generacionales, que nos permiten hablar del arroz con pollo y el sancocho, en la gastronomía, y del tamborito como danza folklórica, este último nos caracteriza a nivel mundial, ni qué decir de la pollera (De la Guardia, 1976; Jaén Suárez, 1979).

La historia nacional inicia con la llegada de los españoles, continúa con nuestra unión a la Colombia pensada por Simón Bolívar y culmina con la separación y creación final de lo panameño en 1903 (todavía estamos escribiendo nuestra historia).

En las historias oficiales se hace referencia a los chinos en la construcción del Ferrocarril transcontinental de Panamá, con ribetes más a lo anecdótico que en la misión de engrandecer su labor; tal vez, por eso minimizan a los chinos con suicidios masivos, uso de opio y con aquello de que debajo de cada durmiente del ferrocarril yace uno enterrado (Chong Ruíz, 1993). No conozco de ningún arqueólogo local o foráneo que de fe de ese comentario; sin embargo, los chinos contribuyeron a la construcción del primer paso expedito por Panamá en forma de vía férrea. Se les menciona, nuevamente, en los canales francés y norteamericano y que, al momento de la separación, en 1903, un chino de nombre Wong Kong Yee, muere producto de una bala de cañón disparada por los colombianos al partir de Panamá... Como dijo Jorge Lee en una ocasión, tal vez no fue un héroe nacional, pero lo cierto es que ese momento trascendental, había un chino por las calles de la Ciudad de Panamá (Gutiérrez, 2015).

El silencio histórico se mantiene hasta la segunda mitad del siglo XX, cuando el tema de las migraciones se torna obligado: la USMA introduce en su programa de Historia de Panamá un capítulo dedicado a ellos; escritores nacionales comienzan a publicar obras y videos que rescatan el esfuerzo y tesón de una comunidad que ha ganado un gran sitial en la historia socio política y económica del país Panamá,como es el caso de Tilcia Elena Delgado, Berta Alicia Chen, Ramón Mon, Juan Tam, Andrea Siu, LockSiu, Eustorgio Chong Ruiz, Amado Araúz, Emma Gómez, Diana González, Araceli Masterson, Naomí Pérez, Luis Alberto Picard-Ami, Robespierre Villar, Luis Pulido Ritter, Damaris Santamaría y Alonso Roy. Esto no significa que son los únicos, pero si los más consultados. 
Invest. pens. crit. Vol. 3, No. 1, enero-abril 2015.

pp. $39-46$

Sin embargo, para lograr esa integración, de la cual el Estado Panameño hoy está muy orgullo, considero que debo hacer un repaso, si bien apretado, por lo menos conciso, sobre los movimientos poblacionales del Istmo de Panamá y así entender parte de la actitud de los panameños de 1903 a 1946, con respecto a las minorías étnicas, religiosas y raciales.

De conformidad a los últimos datos arrojados, gracias a las excavaciones del tercer juego de esclusas, el istmo de Panamá se formó hace unos 25 millones de año y no tres como nos habían notificado nuestros vecinos del Smithsonian (EFE, 2011).

Si las observaciones de Marvin Harris (1968) son correctas, el hombre procedente de Asia pasó en cuatro momentos por Bering. Quiero exagerar y pensar, que los primeros proto-chinos comienzan a ocupar la parte norte del continente americano hará cosa de unos 38 a 40 mil años de antigüedad, lo siento por el señor Colón.

Descendientes de esos primeros hombres que entraron por Bering los encontramos en el Istmo de Panamá hace unos 9 mil años antes de Cristo y para los que prefieren emplear la línea recta hace unos 11 mil años B.P., eran cazadores de megafauna, que evolucionaron como agricultores, ceramistas, orfebres, escultores, constructores (Harris, 1968; Jaén Suárez, 1981).

Estos son los verdaderos pueblos originarios que fueron llevados a la extinción, primero por los ngäbes, bugleres, emberaes, wounaan, gunas, teribes nasos, bribries (quiénes se hacen llamar pueblos originarios), y luego por los españoles y sus asociados negros africanos, estos últimos llegaron en calidad de esclavos, pero su presencia no es tan influyente, salvo en el folklore, como la de los negros libres que llegaron del Caribe contratados como mano de obra en el siglo XIX, cuyos descendientes comienzan a influir en el desarrollo cultural de Panamá hasta el día de hoy (Jaén Suárez, 1981).

Con España, el Istmo de Panamá se transforma en el primer sitio de tierra firme donde las operaciones de expansión imperial se ponen de manifiesto: se funda la Ciudad de Santa María La Antigua del Darién; se llega al Mar del Sur; se fundan las ciudades de Nombre de Dios y de Panamá, definiendo la primera ruta de comercio interoceánica de América, perfeccionándose con la fundación de la ciudad de Portobello, la que se mantendrá hasta la construcción del ferrocarril Panamá-Colón (1855). Todo el comercio favoreció al istmo de Panamá, al extremo de que España se hacía de la vista gorda en lo relativo al contrabando. Con las guerras suramericanas de independencia, el comercio floreció más, dado que los únicos puertos no afectados por la guerra eran, precisamente, los de Portobello y Panamá (Harris, 1968; Jaén Suárez, 1981).

Para desgracia económica de los istmeños-panameños, cuando todo parecía marchar bien, la guerra alcanzó al Istmo y no se tuvo otra opción que arroparse con la bandera de la independencia, pero no la propia, sino la del proyecto Bolívar-Santander, llamado Colombia. 
Invest. pens. crit.

Vol. 3, No. 1, enero-abril 2015.

pp. $39-46$

Bolívar no nos da cabida en su proyecto como un igual y nos traslada, como Departamento, a la soberanía y Jurisdicción de la Nueva Granada. Nombra un Jefe militar venezolano, nos quita territorios, organiza un Congreso Anfictiónico al que no vino; pero, económicamente, no nos fue tan mal. Nada que hacer... Sin embargo, el siglo XIX, unidos a la Nueva Granada como Departamento, nos permite consolidar la mentalidad mercantilista del territorio y todo ello queda evidenciado en la separación de 1830, 1831, el intento de República de 1840 y la Experiencia Federal de 1855-1885 Jaén Suárez, 1981; Castillero R., 1982).

Es en éste período llamado colombiano o de unión voluntaria a Colombia, donde se construye el ferrocarril y el ambiente socio-económico-racial comienza a cambiar el rostro del Istmo de Panamá: llegan europeos y americanos de religión cristiana y judía, indostanes de fe brahamánica e islámica, negros de casi todo el caribe y chinos..., los primeros chinos, que de acuerdo al Panamá Herald, arribaron el 30 de marzo de 1854, a bordo del Bergantín Sea Witch (Chong, 1973; Mon, 1979).

Juan Tam, en una publicación especial de la Revista Lotería, dice que entre "1852 y 1856, se estima que entraron a Panamá a través de las agencias en Hong Kong y Macao, unos 20,000 trabajadores chinos." Pero agrega, rápidamente, que "Estas cifras no indican si realmente llegó esta cantidad de emigrante...”. De ser demostrado esto que dice Tam (2005), tendríamos que mover la fecha que estamos celebrando hoy.

Ramón Mon (2005) ve ese momento como la primera oleada y agrega la existencia de una segunda oleada migratoria que comprende "entre la terminación de los trabajos del ferrocarril y los inicios de la construcción del Canal de Panamá por los norteamericanos", existen otras oleadas migratoria, pero estas tendrán que ver con las oportunidades de hacer negocios, de crecer la familia y de vivir en paz en Panamá (Tam, 2005; Lewis, 2014).

\section{Todo marchaba bien.}

Las minorías no eran amenazas para los istmeños locales cuando éramos colombianos. Las comunidades foráneas no representaban amenaza ni competencia económica. Solo q los chinos en el siglo XIX le aparece una sociedad antichina, a más nadie: solo a los Chinos.

Las leyes protegía los negocios localesy cuando el comercio parecía languidecer, por los errores franceses en el proyecto canalero, la entrada de Estados Unidos fortaleció la fe en la prosperidad de Panamá. Pero Colombia no aceptó el Tratado de 99 años conocido como Herrán-Hay, por ser ofensivo a su soberanía nacional. Los panameños veían zozobrar sus aspiraciones librecambistas y sus intereses llegan a coincidir con el de los norteamericanos, y aprovechan la situación. Se separan de Colombia. Firman con los Estados Unidos el Tratado Hay-Bunau-Varilla, de 18 de noviembre de 1903 y se inicia, lo que Julio Yau llamó El Calvario de un Pueblo (Yau, 1974). Pese a ese calvario la economía se fortaleció y se dolarizó. Y las minorías comenzaron a preocupar a los legisladores y gobernantes y la pureza se volvió, asimismo, una prioridad. 
Lo irónico de todo esto es que el responsable histórico del asunto racial parece ser Arnulfo Arias Madrid. La memoria es tan corta, que nadie parece recordar que durante la Presidencia de Manuel Amador Guerrero y Belisario Porras se dieron leyes excluyentes más severas que la norma constitucional de 1941 (Chen P., 2005).

Veamos brevemente:

Desde la construcción del Ferrocarril de Panamá y el canal Francés, la repatriación de la mano de obra no fue eficiente. El fracaso de los franceses dejó varado en Panamá muchos inmigrantes de oriente y del Caribe; algunos chinos migraron hacia el Caribe o los Estados Unidos; otros, desde la perspectiva de la nueva identidad panameña, comenzaron a competir con los comerciantes locales en lo relativo al negocio al por menor.

La presión de los migrantes fue tal, que durante la construcción del canal por parte de los Estados Unidos, aparecen barrios como El Chorrillo, Calidonia, El Marañón, San Miguel, Malambo y otros en la Ciudad de Colón (Bamboo Line), y en todos había chinos.

La cadena de leyes y Decretos discriminantes contra los chinos y otras etnias, se inició en fecha temprana (Chen P., 2005):

- 1904, Ley 6 de 25 de abril, pone cortapisas a los nacidos en Asia, las Antillas, chinos, mongólicos, semíticos turcos y sirios.

- 1909, Decreto N42 de 24 de junio, ordena la suspensión de la inscripción en las Municipalidades de la República, y el otorgamiento de Cartas de ciudadanía a favor de los chinos, sirios y turcos residentes en el país.

- 1912, se emitieron Decretos que desarrollan e implementan los actos jurídicos antes mencionados.

- 1913 se dicta la Ley 50 de 24 de marzo, es el período de Belisario Porras, período donde aparecen leyes de civilización de indígenas que nos llevaron a una guerra en 1925.

- 1926, Ley 13, prohibió expresamente la inmigración de los chinos, japoneses, sirios, turcos, índico-orientales, indo-arios, dravinianos, negros de las Antillas y negros de las Guyanas.

- 1928, Ley 6, aceptaba un cupo de 10 personas por año para las migraciones prohibidas.

- 1931, Decreto N43 del 27 de mayo, época del Movimiento de Acción Comunal, toleraba a los europeos, pero no así las migraciones procedentes de África.

- 1932, Ley 26 de diciembre, donde se insistía en la prohibición de ingreso al país de chinos, libaneses, palestinos, sirios, turcos y negros, que no tuvieran como idioma materno el castellano.

- 1934, Ley 46 del 24 de diciembre, corrigió la Ley anterior e incluyó a los indostanes.

Todo estos antecedentes jurídicos los cargó, los colocó y lo recalcaron los constituyentes en la constitución de 1941.Empero, la figura ascendente del Doctor Arnulfo Arias Madrid lo hizo amado y 
Invest. pens. crit.

Vol. 3, No. 1, enero-abril 2015.

pp. 39-46

odiado, por sus seguidores y detractores. Se aduce que desde 1934, Arnulfo Arias Madrid, tenía una actitud hitleriana y que publicó un folleto titulado Mejoramiento de la raza, que a decir de sus adversarios, fue introducido en la Constitución del 41, como razas de inmigración prohibida. Esto todavía no se ha demostrado.

Mariela Sagel (2010), cita un documento donde se afirma la entrada al país de "razas indeseables", y de "una nube negra de habla inglesa" que estaban en "los barrios de Las Sabanas, Pueblo Nuevo y Río Abajo", igualmente, "una mancha amarilla se extiende por las aldeas, pueblos y ciudades 'que arrebatan con sus métodos comerciales de cuartillo y su dieta de arroz y chopsuey los negocios de las manos de los panameños".

La Constitución de 1941 fue aprobada por la Asamblea mediante Acto Legislativo de 2 de noviembre de 1940. Ese fue el momento para detenerla, pero se aprobó mayoritariamente.

Debemos entender que la acción de Arnulfo Arias Madrid, en lo relativo a las migraciones a suelo panameño, contemplado en el Artículo 23, obedeció a ese rosario de disposiciones legales que citadas previamente: "Son de inmigración prohibida: la raza negra cuyo idioma originario no sea el castellano, la raza amarilla y las razas originarias de la India, el Asia Menor y el Norte de África” (íbidem).

En definitiva, Arias, tampoco podía saber, como escribe Ramón Mon (2005), sobre La migración china en Panamá: “... si el pueblo en general era adverso a los chinos, turcos y sirios como grupo étnico, pero era obvio que en la mente de algunos legisladores anidaban groseros prejuicios étnicos".

Con estos antecedentes, algunos muy próximos y otros lejanos, ¿cómo podría el legislador y el Ejecutivo, encabezado por Arnulfo, no incluir el aspecto de las inmigraciones prohibidas en la Constitución del 41? De todas formas el peso histórico cayó en la figura de Arias Madrid, quien no pudo estar del todo exento, en materia migratoria, como lo revela su discurso de investidura presidencial del $1^{\circ}$ de octubre de 1940 (Arias Madrid, 1940/1988). Arnulfo es derrocado en 1941 y a pesar de todas las críticas, la constitución del 41 estuvo vigente hasta 1946. Tal vez si no se conocieran las atrocidades de la pretendida supremacía aria en la Segunda Guerra Mundial, la constitución estaría vigente un par de años más.

\section{¿Por qué se asimilaron los chinos y otros grupos nacionales o de cultura religiosa diferente a la vida nacional?}

Primero, porque muchos ya habían nacido en suelo panameño, otros tenían negocios prósperos y no quedaba más opción que asimilarse o integrarse o irse, por eso la siguiente generación se asimiló y cambió la geografía humana panameña de manera tal que más de un tercio de la población panameña tiene más de chino que de otro componente racial en el país. Los descendientes se hicieron cristianos y adoptaron el castellano como primera lengua y sus abuelos delinearon lo que se conoce como barrio chino. 
Invest. pens. crit. Vol. 3, No. 1, enero-abril 2015. pp. $39-46$

La segunda guerra mundial paró en todo el orbe, la discriminación y, hasta hace poco, en Panamá se dejó de hablar de razas. Por eso los chinos de la presente oleada migratoria, post guerra mundial, no se ven presionados para asimilarse, ni para aprender nuevas costumbres.

$\mathrm{Al}$ igual que los judíos y musulmanes, los chinos y los indostanes forman parte de la cadena de tolerancia, la nueva forma de discriminación impuesta por la cultura dominante, a pesar de la participación activa, en todos los niveles de la vida nacional, de estas culturas y naciones milenarias. Mientras no me incomode tu presencia...puedes estar allí.

\section{Referencias}

Arias Madrid, A. (1934). Eugenesia y el mejoramiento de la raza. Boletín Sanitario de Panamá.

Arias Madrid, A. (1988). Discurso. En R. Soler (Ed.). El pensamiento politico en Panamá en los Siglos XIX y XX (Tomo 6 de Biblioteca de la cultura panameña). Panamá: Universidad de Panamá. (Trabajo original publicado en 1940). Disponible en http://bdigital.binal.ac.pa/bdp/descarga.php?f=pensapo9.pdf

Castillero R., E.J. (1982). Historia de Panamá (8va. Ed.). Panamá: Editora Renovación.

Chen, B.A. (2005). De la China a Panamá. Revista Cultural Lotería, 459, 87-117.

Chen, B.A. (2006). Cómo, cuándo y porqué llegaron los chinos a Panamá. Panamá: Management Development Corp.

Chong Ruíz, E. (1993). Los chinos en la sociedad panameña. Panamá: Instituto Nacional de Cultura, Dirección Nacional de Extensión Cultural, Departamento de Letras.

Chong, M. (1973). Historia de Panamá (2da. Ed.) Panamá: Ediciones Guadalupe.

Constitución de la República de Panamá. (1941). Panamá: Imprenta Nacional.

De la Guardia, R. (1976). Mitología panameña. Panamá: Instituto Nacional de Cultura.

EFE. (24 de junio de 2011) Revelan verdadera edad del istmo de Panamá. ABC Color. Disponible en http://www.abc.com.py/ciencia/revelan-verdadera-edad-del-istmo-de-panama-275156.html

Gutiérrez, V. (20 de marzo de 2015). El vínculo entre China y Panamá. La Prensa. Disponible en http://impresa.prensa.com/vivir/vinculo-China-Panama 0 4174832601.html

Harris, M. (1968). The rise of anthropological theory. Current Antrhopology, 9(5), 519-533.

Jaén Suárez, O. (1979). La población del Istmo de Panamá del siglo XVI al siglo XX. Panamá: Impresora de la Nación. 
Invest. pens. crit.

Vol. 3, No. 1, enero-abril 2015.

pp. $39-46$

Jaén Suárez, O. (1981). Hombres y ecología en Panamá. Panamá: Editorial Universitaria y Smithsonian Tropical Research Institute.

Lewis, E. (8 de marzo de 2014). Comunidad china, historia que comenzó sobre rieles. Panamá América. Disponible en http://www.panamaamerica.com.pa/dia-d/comunidad-china-historia-quecomenzo-sobre-rieles

Mon, R.A. (1979). Historia de la migración china durante la construcción del Ferrocarril de Panamá. México: Centro de Estudios de Asia y África del Norte, El Colegio de México.

Mon, R.A. (2005). Mecanismos de adaptación psicológica y procesos de integración de los inmigrantes chinos. Revista Cultural Lotería, 459, 56-62.

Sagel, M. (2010). El racismo de Arnulfo Arias. El Blog de Mariela Sagel. Disponible en http://marielasagel.com/2010/09/el-racismo-de-arnulfo-arias/

Tam, J. (2005). Huellas chinas en Panamá. Revista Cultural Lotería, 459, 7-45.

Yau, J. (1974). El Canal de Panamá, calvario de un pueblo. Madrid, España: Editorial Mediterráneo. 